Journal Club

Editor's Note: These short reviews of recent JNeurosci articles, written exclusively by students or postdoctoral fellows, summarize the important findings of the paper and provide additional insight and commentary. If the authors of the highlighted article have written a response to the Journal Club, the response can be found by viewing the Journal Club at www.jneurosci.org. For more information on the format, review process, and purpose of Journal Club articles, please see http://www.jneurosci.org/content/ jneurosci-journal-club.

\title{
Paraventricular Thalamus Activity during Motivational Conflict Highlights the Nucleus as a Potential Constituent in the Neurocircuitry of Addiction
}

\author{
๑Lauren D. Hill-Bowen, ${ }^{*}$ ๑) Jessica S. Flannery, ${ }^{\star}$ and $\odot$ Ranjita Poudel ${ }^{\star}$ \\ Department of Psychology, Florida International University, Miami, Florida 33199 \\ Review of Choi et al.
}

The paraventricular thalamus (PVT) is associated with reward-related behaviors, including learning cue-reward associations, attributing incentive salience to stimuli, and performing goal-motivated behaviors (Haight and Flagel, 2014; Hsu et al., 2014). Such behavioral associations are intuitive when considering the PVT's afferent connections from the PFC and lateral hypothalamus and efferent projections to the NAc, subiculum, and amygdala. Evidence suggests that PFC-PVT projections modulate reward- and fearrelated behaviors possibly by maintaining cue-outcome associations. For example, optogenetic activation of glutamatergic PFC neurons that project to the PVT during presentation of reward-associated cues reduces cue-induced responses downstream in the NAc, and this dampens both cue-outcome learning and cue-induced approach behaviors while sparing rewardconsumption behaviors (e.g., drinking sucrose) (Otis et al., 2017). On the other hand, optogenetic inhibition of PVTprojecting PFC glutamatergic neurons during presentation of fear-associated

\footnotetext{
Received Aug. 9, 2019; revised Nov. 25, 2019; accepted Dec. 2, 2019. The authors declare no competing financial interests. *L.D.H.-B., J.S.F., and R.P. contributed equally to this work.

Correspondence should be addressed to Lauren D. Hill-Bowen at Ihill045@fiu.edu.

https://doi.org/10.1523/JNEUROSCl.1945-19.2019

Copyright $\odot 2020$ the authors
}

cues reduces downstream amygdala responses, which can reduce subsequent fear retrieval and reduce the expression of cue-induced avoidance behavior (e.g., freezing) (Do-Monte et al., 2015). As opposed to cue-related processing, GABAergic lateral hypothalamus-PVT projections appear to be involved with relaying information about reward consumption (Otis et al., 2019). These PFC and lateral hypothalamus inputs converge in the PVT, which, in turn, sends projections to other limbic structures. Specifically, an anterior PVT subregion (aPVT) projects to the ventral subiculum and dorsomedial NAc shell, whereas a posterior subregion (pPVT) projects to the extended central amygdala and ventromedial NAc shell. Although the functional distinctions between aPVT and pPVT and their differential projections remain to be more fully characterized, emerging evidence suggests that these subregions process conflicting types of information relevant for motivated behaviors (AlHasani et al., 2015). That is, the aPVT appears to be linked with appetitive and the pPVT with aversive information processing (Do-Monte et al., 2017). Thus, the PVT as a whole may play a role in resolving motivational conflict when an animal encounters complex real-world situations eliciting competing approach and avoidance behaviors.
In a recent article in The Journal of Neuroscience, Choi et al. (2019) examined functional distinctions between these PVT subregions during motivational conflict. To induce such conflict, the authors used a Pavlovian counterconditioning task. In this task, a conditioned stimulus (CS, tone) was first paired with either an appetitive (liquid sucrose solution) unconditioned stimulus (US) in an appetitive context (house light off, solid floor, rose odor) or an aversive (electrical foot shock) US in an aversive context (house light on, grid floor, peppermint odor). After rats learned the first association, the same CS tone was paired with the US and context of the opposite valence (i.e., appetitive-to-aversive or aversive-to-appetitive training). Thus, via training, the CS acquired conflicting motivational value (i.e., both appetitive and aversive associations). During a subsequent testing phase, each rat received one presentation of the CS tone in each context (appetitive and aversive). $\mathrm{Ca}^{2+}$ imaging was used to measure aPVT and pPVT subregion activity after CS (tones) and US (sucrose, foot shock) presentations during appetitive and aversive training, as well as during appetitive and aversive testing phases.

During the appetitive phase of appetitiveto-aversive counterconditioning training, CS tone presentation elicited a significant (yet variable) aPVT response that was not 
modulated by the number of training sessions encountered. The aPVT was not responsive to US sucrose-reward consumption. In contrast, pPVT responded to both CS tone presentation and US reward consumption, and responsivity to both increased over the course of training. During the subsequent aversive training phase, aPVT responded weakly to the CS tone (reaching significance only late in training) but responded robustly to US shock. In contrast, pPVT responded robustly to both the CS tone and US shock across all trials. Correlational analyses between tone-elicited PVT activity and reward-approach behavior identified aPVT activity as a weak, yet significant, predictor and pPVT activity as a robust predictor of reward approach behavior.

During the aversive phase of aversiveto-appetitive counterconditioning, aPVT and pPVT activity patterns were similar to those described above. But only pPVT activity was a significant predictor of defensive freezing. In sum, aPVT responses were distinctly linked to reward-approach behavior, and pPVT responses were linked to both reward-approach and shockavoidance behaviors.

During subsequent testing, approach and avoidance behaviors differed based on the counterconditioning paradigm (i.e., appetitive-to-aversive vs aversive-toappetitive). When appetitive training occurred first, rats were more likely to exhibit CS-induced reward-approach behaviors in the appetitive context and to exhibit more shock-avoidance behaviors during testing in the aversive context. Conversely, when aversive training occurred first, there was no evidence for context-dependent behavioral differences during testing in either the appetitive or aversive contexts. Across counterconditioning paradigms, aPVT and pPVT responded to CS tone presentation in both the appetitive and aversive contexts, with no differences in magnitude of these signals across contexts.

To then provide evidence that PVT exerted a causal influence on behavior during motivational conflict, Choi et al. (2019) used a designer receptor exclusively activated by designer drugs technique to inhibit either the aPVT or pPVT, or both subregions before testing in each counterconditioning paradigm. Inhibition of the entire PVT, but not the aPVT or pPVT alone, disrupted reward-approach behaviors and increased shock-avoidance behaviors in both counterconditioning paradigms during testing. These outcomes suggest that the combined activity of both PVT subregions was necessary for context-appropriate approach and avoidance behavior in the presence of conflict. To provide confirmatory evidence of this interpretation, the authors considered the effect of designer receptor exclusively activated by designer drugsmediated inhibition of the entire PVT on appetitive and aversive behaviors in the absence of conflict using a different cohort of rats. That is, rats were exposed only to aversive or appetitive conditioning (as opposed to both) before testing. In these experiments, PVT inhibition did not affect reward-approach or shock-avoidance behaviors, implying that motivational conflict is an important perquisite for PVT-related influences on behavior.

Together, these results suggest that the PVT is responsive to cues that predict reward or danger during appetitive and aversive conditioning and plays a prominent role in an animal's ability to use learned information for executing contextappropriate behaviors. The authors speculate that, in the test trials, the differential PVT responsivity following appetitive and aversive conditioning along an anteriorposterior axis is linked with the aPVT's and pPVT's distinct projections to the dorsomedial and ventromedial NAc shells, respectively. This perspective is supported by evidence indicating that optogenetic stimulation of dorsomedial NAc shell elicits approach behaviors (i.e., place preference) and stimulation of ventromedial NAc shell elicits avoidance behaviors (i.e., dysphoria) (Al-Hasani et al., 2015). Consistent with this observation, a greater number of neurons in the pPVT (vs aPVT) send collateral efferents to the lateral and central amygdala, nuclei commonly implicated in avoidance behaviors (Dong et al., 2017).

The results of Choi et al. (2019) further implicate PVT in regulating behavior during motivational conflict. Such behavioral regulation during conflict potentially stems from the PVT's ability to modulate dopamine release in the NAc. Specifically, PVT electrical stimulation increases extracellular dopamine levels in the NAc shell, and activation of the PVT-NAc pathway increases spontaneous DAergic cell firing in the VTA (Parsons et al., 2007; Perez and Lodge, 2018). NAc neurons expressing dopamine receptors have been consistently implicated in attributing incentive salience to reward cues (Saunders and Robinson, 2012) and resolving motivational conflict (Piantadosi et al., 2017; Nguyen et al., 2018). Therefore, modulation of dopamine release in the NAc is a potential mechanism by which PVT activ- ity influences behavior in situations eliciting competing approach and avoidance behaviors.

Dysregulated DAergic functioning is a common feature of addiction; and given the existing link between PVT activity and NAc dopamine levels, the PVT has been increasingly recognized as a constituent in the neurocircuitry of addiction (Di Chiara and Bassareo, 2007; Parsons et al., 2007). Maladaptive resolution of conflicting approach and avoidance associations may contribute to the maintenance of compulsive drug use despite the emergence of negative health, social, and financial consequences, a defining aspect of addiction (e.g., Nguyen et al., 2015). As the motivational value of addictive drugs is disproportionately high and outweighs the value of aversive consequences, repeated drug exposure may disrupt regulation of approach (drug-seeking and taking) and avoidance (drug abstinence) behaviors (Nguyen et al., 2015). Consistent with this perspective, repeated cocaine exposure has been shown to lead to dysregulation of positive and negative motivational processing. Specifically, rats preexposed to cocaine displayed a bias toward appetitive approach when presented with a cue associated with both sucrose reward and shock punishment (Nguyen et al., 2015).

The findings of Choi et al. (2019) may be relevant for interpreting previous studies demonstrating that disruption of PVT functioning dampens context-induced drug-reinstatement behaviors (Hamlin et al., 2009; Pelloux et al., 2018). The counterconditioning paradigm of Choi et al. (2019) mirrors drug reinstatement paradigms, which create motivational conflict by altering the motivational value of a CS (drug-related cue). However, drug reinstatement paradigms may create motivational conflict by first pairing cues/ contexts with drug-reward delivery, and subsequently with no-reward delivery. Context- or cue-induced reinstatement consequently requires resolving conflicting behavioral responses to cues/contexts resulting in the reemergence of the extinguished drug-oriented behavior (Pelloux et al., 2018). Therefore, the Choi et al. (2019) finding regarding the PVT's involvement in CS-induced approach behavior hints at the PVT's involvement in reinstatement behavior and, by extension, cue-induced drug relapse.

The Choi et al. (2019) demonstration of pPVT responsivity to aversive stimuli also has implications for models of addiction. Specifically, pPVT responsivity to both aversive US and respective CS stim- 
uli during cue-learning, and its correlation with defensive freezing behavior, is consistent with the PPVT's projections to the ventromedial NAc shell, a region implicated in avoidance behaviors (Choi et al., 2019). Previous work highlights the potential role of this PVT-NAc pathway in addiction, where disruption thereof reduces withdrawal-induced place aversion and somatic signs of opiate withdrawal (Zhu et al., 2016). The necessity of PVTNAc pathway functioning for drug selfadministration and the aversive effects of withdrawal, but not craving-induced drug-seeking behavior, suggests that this pathway may be involved in drug use motivated by negative reinforcement (Huang et al., 2018). Overall, research investigating the role of PVT function in regulating approach and avoidance behaviors has the potential to shed light on the motivational mechanisms that drive choices between pursuing drug-related behaviors (e.g., drug seeking and taking) and avoiding their respective negative consequences (e.g., health, social, and financial consequences).

\section{References}

Al-Hasani R, McCall JG, Shin G, Gomez AM, Schmitz GP, Bernardi JM, Pyo CO, Park SI, Marcinkiewcz CM, Crowley NA, Krashes MJ, Lowell BB, Kash TL, Rogers JA, Bruchas MR (2015) Distinct subpopulations of nucleus accumbens dynorphin neurons drive aversion and reward. Neuron 87:1063-1077.

Choi EA, Jean-Richard-Dit-Bressel P, Clifford CW, McNally GP (2019) Paraventricular thalamus controls behavior during motivational conflict. J Neurosci 39:4945-4958.

Di Chiara G, Bassareo V (2007) Reward system and addiction: what dopamine does and doesn't do. Curr Opin Pharmacol 7:69-76.

Do-Monte FH, Quiñones-Laracuente K, Quirk GJ (2015) A temporal shift in the circuits mediating retrieval of fear memory. Nature 519:460-463.

Do-Monte FH, Minier-Toribio A, QuiñonesLaracuente K, Medina-Colón EM, Quirk GJ (2017) Thalamic regulation of sucrose seeking during unexpected reward omission. Neuron 94:388-400.e4.

Dong X, Li S, Kirouac GJ (2017) Collateralization of projections from the paraventricular nucleus of the thalamus to the nucleus accumbens, bed nucleus of the stria terminalis, and central nucleus of the amygdala. Brain Struct Funct 222:3927-3943.

Haight JL, Flagel SB (2014) A potential role for the paraventricular nucleus of the thalamus in mediating individual variation in Pavlovian conditioned responses. Front Behav Neurosci 8:79.

Hamlin AS, Clemens KJ, Choi EA, McNally GP (2009) Paraventricular thalamus mediates context-induced reinstatement (renewal) of extinguished reward seeking. Eur J Neurosci 29:802-812.

Hsu DT, Kirouac GJ, Zubieta JK, Bhatnagar S (2014) Contributions of the paraventricular thalamic nucleus in the regulation of stress, motivation, and mood. Front Behav Neurosci 8:73.

Huang AS, Mitchell JA, Haber SN, Alia-Klein N, Goldstein RZ (2018) The thalamus in drug addiction: from rodents to humans. Philos Trans R Soc Lond B Biol Sci 373:20170028.

Nguyen D, Schumacher A, Erb S, Ito R (2015) Aberrant approach-avoidance conflict resolution following repeated cocaine pre-exposure. Psychopharmacology 232:3573-3583.

Nguyen D, Fugariu V, Erb S, Ito R (2018) Dissociable roles of the nucleus accumbens D1 and D2 receptors in regulating cue-elicited approach-avoidance conflict decision-making. Psychopharmacology 235:2233-2244.
Otis JM, Namboodiri VM, Matan AM, Voets ES, Mohorn EP, Kosyk O, McHenry JA, Robinson JE, Resendez SL, Rossi MA, Stuber GD (2017) Prefrontal cortex output circuits guide reward seeking through divergent cue encoding. $\mathrm{Na}$ ture 543:103-107.

Otis JM, Zhu M, Namboodiri VM, Cook CA, Kosyk O, Matan AM, Ying R, Hashikawa Y, Hashikawa K, Trujillo-Pisanty I, Guo J, Ung RL, Rodriguez-Romaguera J, Anton ES, Stuber GD (2019) Paraventricular thalamus projection neurons integrate cortical and hypothalamic signals for cue-reward processing. Neuron 103:423-431.e4.

Parsons MP, Li S, Kirouac GJ (2007) Functional and anatomical connection between the paraventricular nucleus of the thalamus and dopamine fibers of the nucleus accumbens. J Comp Neurol 500:1050-1063.

Pelloux Y, Hoots JK, Cifani C, Adhikary S, Martin J, Minier-Toribio A, Bossert JM,Shaham Y (2018) Context-induced relapse to cocaine seeking after punishment-imposed abstinence is associated with activation of cortical and subcortical brain regions. Addict Biol 23:699-712.

Perez SM, Lodge DJ (2018) Convergent inputs from the hippocampus and thalamus to the nucleus accumbens regulate dopamine neuron activity. J Neurosci 38:10607-10618.

Piantadosi PT, Yeates DC, Wilkins M, Floresco SB (2017) Contributions of basolateral amygdala and nucleus accumbens subregions to mediating motivational conflict during punished reward-seeking. Neurobiol Learn Mem 140: 92-105.

Saunders BT, Robinson TE (2012) The role of dopamine in the accumbens core in the expression of Pavlovian-conditioned responses. Eur J Neurosci 36:2521-2532.

Zhu Y, Wienecke CF, Nachtrab G, Chen X (2016) A thalamic input to the nucleus accumbens mediates opiate dependence. Nature 530:219222. 